\title{
The C677T polymorphism in MTHFR is not associated with migraine in Portugal
}

\author{
Anabela Ferro $^{\mathrm{a}, \mathrm{b}}$, Maria-José Castro ${ }^{\mathrm{a}, \mathrm{b}, \mathrm{c}}$, Carolina Lemos ${ }^{\mathrm{a}, \mathrm{b}, \mathrm{c}}$, Mónica Santos $^{\mathrm{b}, \mathrm{d}}$, Alda Sousa ${ }^{\mathrm{a}, \mathrm{b}}$, \\ José Pereira-Monteiro $^{\mathrm{c}}$, Jorge Sequeiros $^{\mathrm{a}, \mathrm{b}}$ and Patrícia Maciel $^{\mathrm{d}, *}$ \\ ${ }^{a}$ UnIGENe-IBMC, Instituto de Biologia Molecular e Celular, Universidade do Porto, Porto, Portugal \\ ${ }^{\mathrm{b}}$ Departamento de Estudo das Populações, ICBAS, Universidade do Porto, Porto, Portugal \\ ${ }^{\mathrm{c}}$ Centro de Estudo de Cefaleias, Hospital Geral Santo António, Porto, Portugal \\ ${ }^{\mathrm{d}}$ Life and Health Sciences Research Institute (ICVS), School of Health Sciences, University of Minho, Braga, \\ Portugal
}

\begin{abstract}
Migraine is a debilitating disorder affecting a large proportion of the population. The effect of methylenetrathydrofolate reductase (GeneID: 4524) polymorphisms in migraine etiology and development has been a theme of great interest. Several populations were evaluated with contradictory results. In this case-control study, we investigated the effect of the C677T polymorphism in $M T H F R$, as a genetic risk factor for migraine, in the Portuguese population. We observed that, overall, there was no significant difference in the frequencies of MTHFR C677T genotypes or of the T-allele among the Portuguese migraineurs when compared to controls. There was also no association of migraine with aura with MTHFR genotypes or with the T-allele, in contrast with previous studies. Regarding the risk of the T-allele homozygote carriers, there was an equal probability to develop migraine with aura over migraine without aura in our patients. Thus, we conclude that the C677T MTHFR polymorphism, responsible for a reduction of the MTHFR activity in folate metabolism, is not a major genetic susceptibility factor for migraine in the Portuguese population.
\end{abstract}

Keywords: Migraine with aura, association study, folate metabolism, homocysteine, cephalalgia

Abbreviations: MA, migraine with aura; MO, migraine without aura; Hcy, homocysteine; TGV, trigeminovascular system.

\section{Introduction}

Migraine is a common neurovascular disease affecting all age groups but mainly adolescents and young adults. Along with the characteristic episodic headaches, patients often experience nausea, vomiting, photophobia and/or phonophobia. According to the International Headache Society (IHS), there are two major classes of migraine: migraine with aura (MA) and migraine without aura (MO) [1]. The aura symptoms

\footnotetext{
*Corresponding author: Patricia Maciel, Life and Health Sciences Research Institute (ICVS), School of Health Sciences, University of Minho, Campus de Gualtar, 4710-057 Braga, Portugal. Tel.: +351 2536048 24; Fax: +351 2536048 20; E-mail: pmaciel@ecsaude.uminho.pt.
}

precede the head pain, when present, in MA patients, and they usually correspond to visual or sensory disturbances [2].

Several studies were performed in order to define the neuroanatomical involvement in migraine, to clarify its molecular mechanisms and to define the modulating factors influencing this neurovascular disorder. A large set of data supports the concept that migraine is partially associated with cerebral blood circulation disturbances [3].

Increased levels of homocysteine (Hcy), a precursor of excitatory amino acids, have been associated, among others, with vascular integrity [4]. The level of Hcy also affects the trigeminovascular system (TGV) by increasing its firing rate as in response to pain. Since the TGV has been strongly implicated in migraine [5], 
the excitatory effect of Hcy may affect the threshold for migraine manifestation [4].

In humans, the total level of Hcy is determined both by environmental and genetic factors [6]. The enzyme methylenetetrahydrofolate reductase (MTHFR, EC 1.5.1.20) is involved in folate metabolism, catalyzing the reduction of 5-10 methyltetrahydrofolate to 5-methyltetrahydrofolate, an essential substrate for the methylation of Hcy to methionine. Frosst et al. [6] reported an association between a sequence variation in the MTHFR gene (C677T) and plasma Hcy levels. The common C677T substitution in MTHFR results in an aminoacid replacement $(\mathrm{A} 222 \mathrm{~V})$ in the catalytic domain that leads to increased enzyme thermolability and concomitant activity reduction [6]. When dietary folate intake is insufficient, the polymorphism-associated reduction in enzyme activity may result in mild hyperhomocysteinemia [6]. For this reason, the effect of MTHFR polymorphisms on the risk of developing migraine has been a subject of great interest and migraineurs of several populations have been evaluated for this matter [7-13]. In this study, we performed a case-control study to estimate the contribution of the MTHFR C677T polymorphism to the risk of developing migraine in the Portuguese population.

\section{Material and methods}

\subsection{Subjects}

One hundred and eighty-six Portuguese, unrelated migraine patients, $82 \%$ of whom females, with a mean age of $36.2 \pm 11.6$ years, were examined and interviewed by an experienced neurologist (J. P.-M.) between 2000 and 2003. These patients were clinically diagnosed, based on the 1988 IHS criteria [1], as having MA (78 cases, $42 \%$, mean age $34.9 \pm 14.4$ years), or MO (108 cases, $58 \%$, mean age $36.4 \pm 12.3$ years). The patients experiencing both subtypes of migraine (22 cases, 12\%) were included in the MA group. Patients with hemiplegic migraine were excluded from this study. In the patients group, $161(87 \%)$ had clinical history of familial migraine. Informed consent was obtained from all patients and the ethics committee of the Santo António Hospital approved the study. To establish a control population that would be representative of the whole country, 50 Guthrie samples per each of the 20 Portuguese districts were anonymously, randomly and blindly selected from the national program of phenylketonuria, congenital hypothyroidism and other metabolic disordres screening (Guthrie blood spots) biobank (kindly provided by Dr Laura Vilarinho, IGM). Since the migraine patient group was mainly from the North of Portugal, all the Guthrie samples collected from six districts comprising this sub-region (Viana do Castelo, Braga, Porto, Vila Real, Bragança, Aveiro) were genotyped. For the remaining 14 districts, the number of Guthrie card controls genotyped was based on the population frequency of the district in the global Portuguese population. Taken this, a control group ("Guthrie card controls") was establish and encompasses 397 anonymous samples of similar ethnic background to that of the patients. We also genotyped 96 additional controls (blood donors) in whom migraine was excluded and were matched for sex and ethnic background to that of the patient sample. Though a direct age matching was not possible, the samples of the blood donor group have a higher mean age (40.7 \pm $10.8)$ of that of the patient's group $(34.9 \pm 12.7)$.

\subsection{Genotyping}

The genomic DNA of patients and blood controls was extracted from peripheral blood using standard methods. The genomic DNA from Guthrie card controls was extracted from the filter paper blots using Chelex in a final concentration of $0.8 \%$. Amplification of the fragment comprising the MTHFR C677T polymorphism was carried out by PCR [6] using SEQ-MTHFRE4 forward (5'-ACTGTCATCCCTATTGGCAGGTT-3') and MTHFRE4B reverse (5'-AGGACGGTGCGGTGAGA GTGG-3') primers. In the restriction analysis, PCR products were digested with HinfI and then resolved in an $8 \%$ non-denaturing polyacrylamide gel, stained with ethidium bromide and scanned with TYPHOON 8600 (GE Healthcare). Genotype comparisons were made using a 25 pb DNA marker. Genotyping of the MTHFR C677T polymorphism was performed by a researcher who was unaware of the patient's phenotype.

\subsection{Statistical analysis}

The frequency of MTHFR alleles and genotypes were calculated using the SPSS program (version 14). Hardy-Weinberg equilibrium was tested for migraineurs and control groups. Group comparison was made with chi-square test and the level of significance was set at $P<0.05$. The risk of migraine for carriers of the different MTHFR genotypes was assessed by calculating the odds ratios (OR) with $95 \%$ confidence intervals (CI). 


\section{Results}

A group of 186 unrelated migraineurs was compared with two control groups of Portuguese origin: the Guthrie card controls comprising 397 individuals and the blood donor controls $(n=96)$ including samples from non-migraineurs individuals. The allele and genotype frequencies of MTHFR observed in migraine patients and in controls are summarized in Table 1. The genotype frequencies of patients and both control groups were in Hardy-Weinberg equilibrium (migraineurs: $\chi^{2}=2.06$, $1 \mathrm{df}, P=0.15$; Guthrie card controls: $\chi^{2}=0.37,1 \mathrm{df}, P=0.54$; blood donor controls: $\chi^{2}=0.08,1 \mathrm{df}, P=0.78$ ). When comparing the distribution of CC, CT and TT genotype frequencies in the migraine group in general (migraineurs, $42 \%, 49 \%$ and $9 \%$ ) with the ones from the Guthrie card control group (44\%, 44\% and $13 \%)$ we concluded that they were not significantly different $\left(\chi^{2}=2.54\right.$, $2 \mathrm{df} ; P=0.28)$. When the comparison was made with the genotype frequencies in the control population excluded for migraine (blood donor control group, 36\%, $49 \%$ and $15 \%$ ), again, no significant differences were observed ( $\chi^{2}=2.69,2 \mathrm{df} ; P=0.26$ ) (Table 1$)$. The direct comparison of TT genotype frequencies in the two control populations also showed no statistical differences $\left(\chi^{2}=1.62,2 \mathrm{df} ; P=0.45\right)$. When we compared the frequency of T677T homozygote carriers in the migraine sub-groups, MA $(6 \%)$, MO (10\%) and in controls $(13 \%$, Guthrie card controls or $15 \%$ in blood donor controls), again, no significant differences were observed $\left(\chi^{2}=2.65,2 \mathrm{df} ; P=0.26\right.$ and $\chi^{2}=3.06$, 2 df; $P=0.22$, respectively) (Table 1 ). Consistently, no enrichment of the risk T-allele was observed in the migraineurs compared to the controls (Table 1). Direct comparisons of TT homozygosity or risk T-allele frequencies in MA versus $\mathrm{MO}$ or controls showed no significant differences, indicating that no higher risk for developing MA is associated with the T-allele (Table 1). In the MA sub-group we found that the T-allele was less represented (29\%) than in both control groups (35\% in Guthrie card controls or $39 \%$ in blood donor group), although with no statistical significance $\left(\chi^{2}=\right.$ 1.47 , $1 \mathrm{df} ; P=0.22$ or $\chi^{2}=3.48$, $1 \mathrm{df} ; P=0.062$ ), respectively.

\section{Discussion}

Several studies have been performed to evaluate the contribution of the C677T polymorphism in the
MTHFR gene to the risk of developing migraine (summary in Table 2). Kowa et al. [8] reported an association of the TT-genotype with migraine in Japanese MA patients. Kara et al. [7], observed an association of the T-allele in homozygosity with the migraine, overall, but failed to detect this association in Turkish MA patients, in particular. Oterino et al. [10] found no association of the TT-genotype in Spanish migraineurs overall, but a two-fold risk of MA was reported to be associated with the T-allele among the patients. Lea et al. [9] observed, in a case-control study of unrelated patients, a significant association of the TT-genotype with MA in the sporadic migraineurs, as well as in the general unrelated migraine group. Scher et al. [14] found, in a population-based study, a significant increase in the frequency of the TT-genotype among Dutch MA sufferers when compared to controls and two large independent studies reported no involvement of TT-genotype in MA etiology for German [12] and Finnish [13] patients. The same positive association was reported for MA in familial migraineurs when compared to unrelated healthy controls [9]. Population stratification and inappropriate control selection being major confounders in association studies, it is important to notice that in both family based case-control studies reported to date, using a study design that is less sensitive to these issues, the T-allele did not co-segregate preferentially with migraine $[9,12]$. Consistently, Bottini et al. [11] reported no association between this polymorphism in MTHFR and migraine, or its sub-groups, in an Italian prospective study. More recently, a meta-analysis combining all studies assessing the association of MTHFR C677T polymorphism with migraine further suggests that the TT-genotype is a genetic risk factor for MA, but not to migraine overall [15].

In this case-control study, performed in the Portuguese population, we did not observe an increased susceptibility of the heterozygous or homozygous MTHFR C677T carriers to migraine.

Moreover, a stratified analysis of the patients sample into MA and MO sub-groups also did not show an increased risk for experiencing migraine with aura among the patients with this genotype. We actually observed an inverse proportion of the risk T-allele between the MA sub-group and the controls, with subrepresentation of the T-allele in MA, although this was not statistically significant; this same trend has been observed by Todt et al. in the German migraineurs [12].

These results do not completely rule out the possibility of an association between this genetic polymorphism and migraine in our population: alone, this poly- 


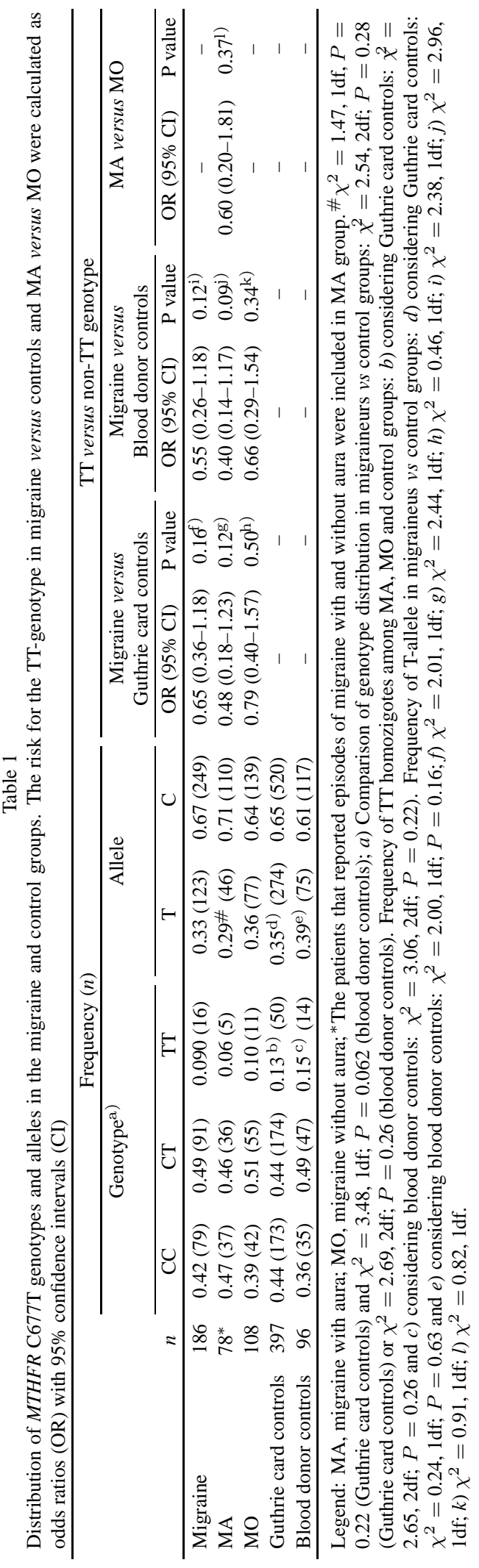




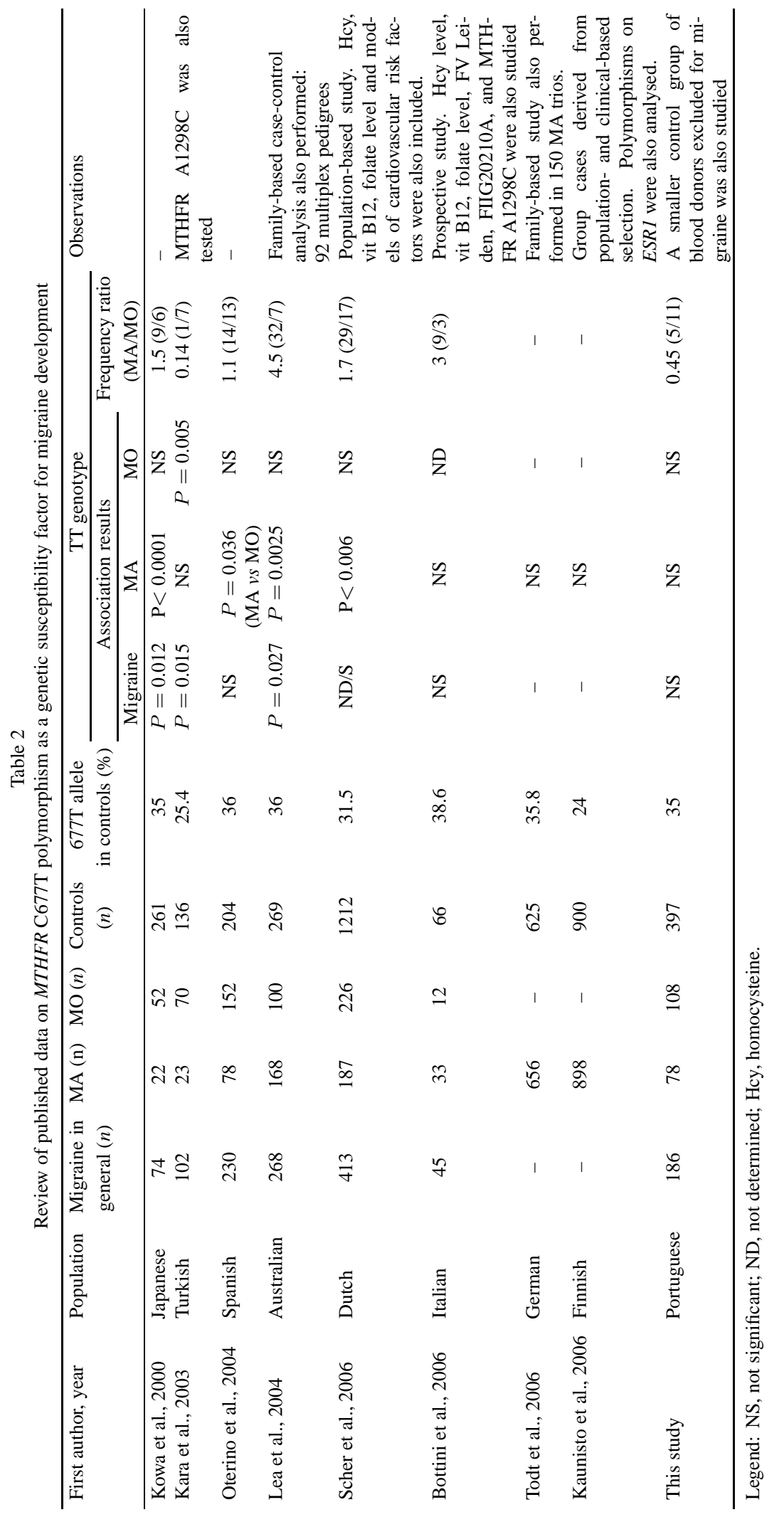


morphism does not seem to confer an increased risk to migraine in the Portuguese population, but it may do so, synergistically, with other genetic or environmental risk factors, which have not been evaluated yet. Nevertheless, we believe that our data is sufficient to exclude a major effect of this polymorphism in the causality of migraine in this population and to conclude that it cannot be used on its own as a predictive factor, either to measure the risk of migraine or to predict the clinical presentation of the disease in affected individuals.

The T-allele frequencies detected in our control samples (35\% in Guthrie card group and 39\% in the blood donor group) are consistent with other studies in Caucasian populations [9], indicating that both these control panels are appropriate ones, that may also be useful for further studies. The fact that other studies with smaller and modest sample populations were able to observe association, when present, between MTHFR C677T and migraine, makes us believe that the size of our migraine population is not the major factor contributing (negatively) to the overall outcome of this study. No statistical difference was observed between the two control groups studied and, although smaller, the blood donor group comprised individuals excluded for migraine. Additionally, we have no reason to expect that the larger control group (Guthrie card controls), although not matched for age and sex with the patient population, nor excluded for migraine could be significantly enriched in migraineurs, since it was randomly selected, thus not biasing the lack of association observed. Interestingly, our study and the Spanish study did not report an association of the TT genotype with migraine and MA, when comparing to controls. The common overall outcome may be a reflection of the genetic proximity reported for the two populations [16]. Further studies concerning other known MTHFR polymorphisms, genetic prothrombotic factors, and/or biochemical profiles, need to be carried out in the Portuguese migraine patients in order to better understand the gene-gene and gene-environment interactions and improve our knowledge of the pathogenesis of migraine.

\section{Acknowledgements}

We thank all participants of this study and Laura Vilarinho, Instituto de Genética Médica, Porto. The study was funded by Fundação para a Ciência e Tecnologia (POCTI-034390/99/FCT) and A.F. (SFRH/BD/ 1288/2000), M.-J.C. (POCTI-034390-PR332009-BTI), M.S. (SFRH/BD/9111/2002), C.L. (SFRH/BD/17761/ 2004) were scholarship recipients from FCT.

\section{References}

[1] Headache Classification Committee of the International Headache Society: Classification and diagnostic criteria for headache disorders, cranial neuralgias and facial pain., Cephalalgia 8 (1988), 1-96.

[2] B.K. Rasmussen and J. Olesen, Migraine with aura and migraine without aura: an epidemiological study, Cephalalgia 12 (1992), 221-228.

[3] E.L. Spierings, Pathogenesis of the migraine attack, Clin J Pain 19 (2003), 255-262.

[4] T. Bottiglieri, Homocysteine and folate metabolism in depression, Prog Neuropsychopharmacol Biol Psychiatry 29 (2005), 1103-1112.

[5] P.J. Goadsby, Y.E. Knight, K.L. Hoskin and P. Butler, Stimulation of an intracranial trigeminally-innervated structure selectively increases cerebral blood flow, Brain Res 751 (1997), 247-252.

[6] P. Frosst, H.J. Blom, R. Milos, P. Goyette, C.A. Sheppard, R.G. Matthews, G.J. Boers, M. den Heijer, L.A. Kluijtmans and L.P. van den Heuvel, A candidate genetic risk factor for vascular disease: a common mutation in methylenetetrahydrofolate reductase, Nat Genet 10 (1995), 111-113.

[7] I. Kara, A. Sazci, E. Ergul, G. Kaya and G. Kilic, Association of the C677T and A1298C polymorphisms in the 5,10 methylenetetrahydrofolate reductase gene in patients with migraine risk, Mol Brain Res 111 (2003), 84-90.

[8] H. Kowa, K. Yasui, T. Takeshima, K. Urakami, F. Sakai and K. Nakashima, The homozygous C677T mutation in the methylenetetrahydrofolate reductase gene is a genetic risk factor for migraine, Am J Med Genet 96 (2000), 762-764.

[9] R.A. Lea, M. Ovcaric, J. Sundholm, J. Macmillan and L.R. Griffiths, The methylenetetrahydrofolate reductase gene variant C677T influences susceptibility to migraine with aura, BMC Med 2 (2004), 3.

[10] A. Oterino, N. Valle, Y. Bravo, P. Munoz, P. Sanchez-Velasco, C. Ruiz-Alegria, J. Castillo, F. Leyva-Cobian, A. Vadillo and J. Pascual, MTHFR T677 homozygosis influences the presence of aura in migraineurs, Cephalalgia 24 (2004), 491-494.

[11] F. Bottini, M.E. Celle, M.G. Calevo, S. Amato, G. Minniti, L. Montaldi, D. Di Pasquale, R. Cerone, E. Veneselli and A.C. Molinari, Metabolic and genetic risk factors for migraine in children, Cephalalgia 26 (2006), 731-737.

[12] U. Todt, J. Freudenberg, I. Goebel, C. Netzer, A. Heinze, K. Heinze-Kuhn, H. Gobel and C. Kubisch, MTHFR C677T polymorphism and migraine with aura, Ann Neurol (2006).

[13] M.A. Kaunisto, M. Kallela, E. Hamalainen, R. Kilpikari, H. Havanka, H. Harno, M. Nissila, E. Sako, M. Ilmavirta, J. Liukkonen, H. Teirmaa, O. Tornwall, M. Jussila, J. Terwilliger, M. Farkkila, J. Kaprio, A. Palotie and M. Wessman, Testing of variants of the MTHFR and ESR1 genes in 1798 Finnish individuals fails to confirm the association with migraine with aura, Cephalalgia 26 (2006), 1462-1472.

[14] A.I. Scher, G.M. Terwindt, W.M. Verschuren, M.C. Kruit, H.J. Blom, H. Kowa, R.R. Frants, A.M. van den Maagdenberg, M. van Buchem, M.D. Ferrari and L.J. Launer, Migraine and MTHFR C677T genotype in a population-based sample, Ann Neurol 59 (2006), 372-375.

[15] E. Rubino, M. Ferrero, I. Rainero, E. Binello, G. Vaula and L. Pinessi, Association of the C677T polymorphism in the MTHFR gene with migraine: a meta-analysis, Cephalalgia (2007). 
[16] S. Beleza, L. Gusmão, A. Lopes, C. Alves, I. Gomes, M. Giouzeli, F. Calafell, A. Carracedo and A. Amorim, Micro-
Phyloeographic and Demographic History of Portuguese Male lineages, Ann Hum Genet 70 (2006), 181-194. 


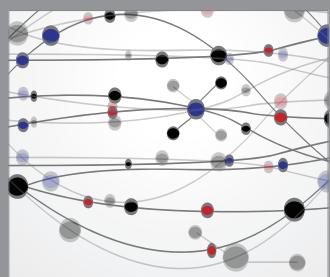

The Scientific World Journal
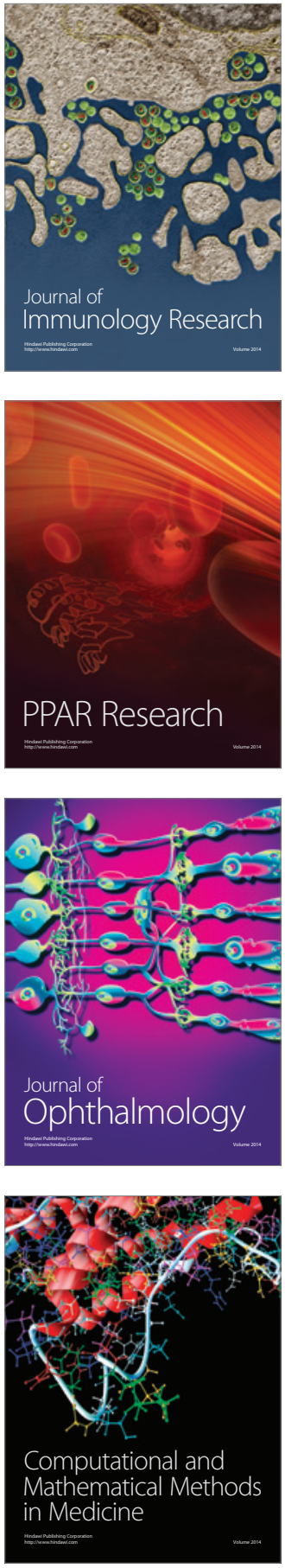

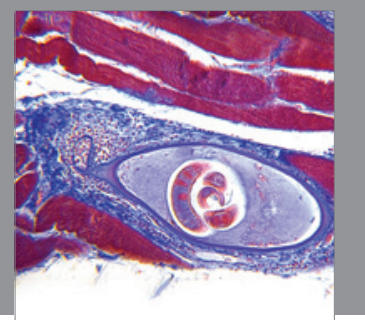

Gastroenterology

Research and Practice
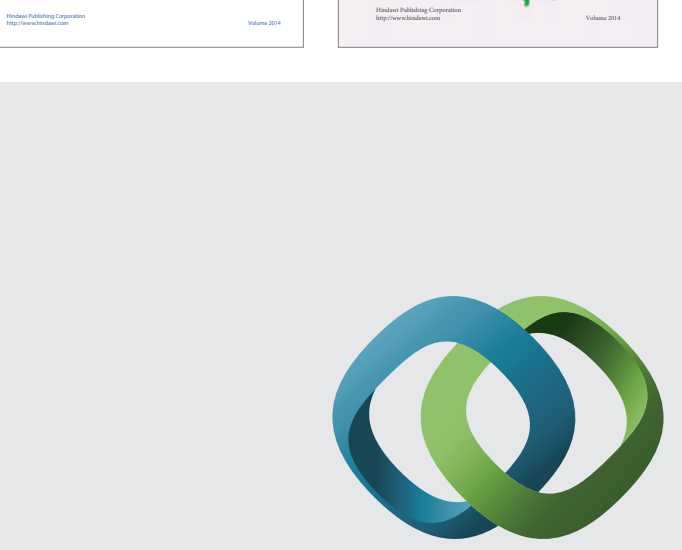

\section{Hindawi}

Submit your manuscripts at

http://www.hindawi.com
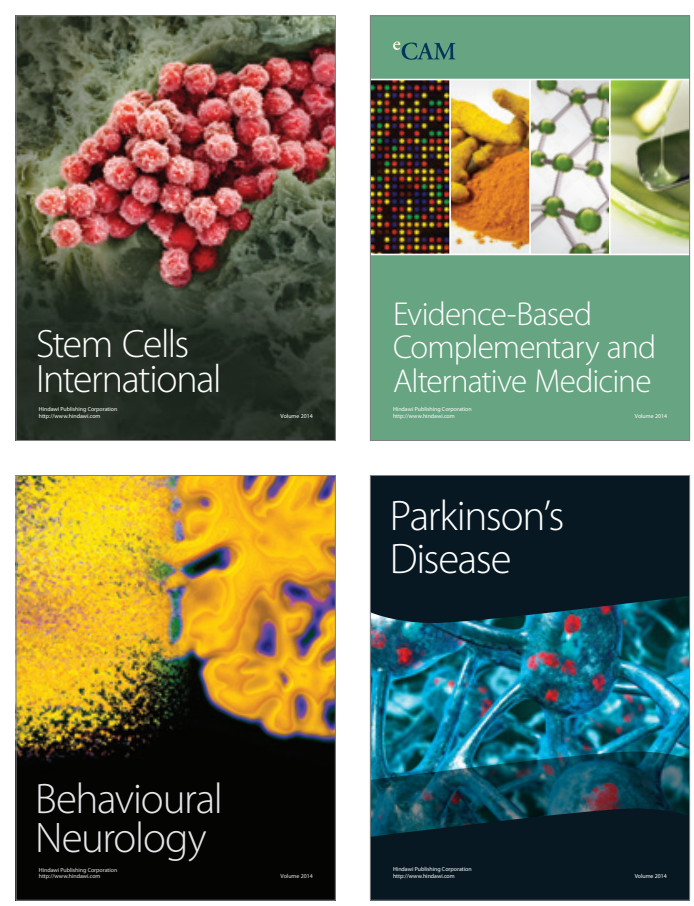

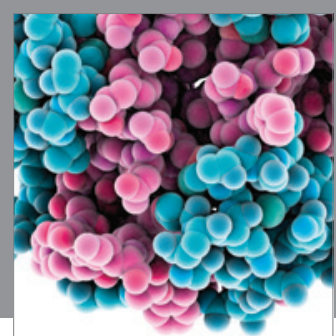

Journal of
Diabetes Research

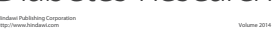

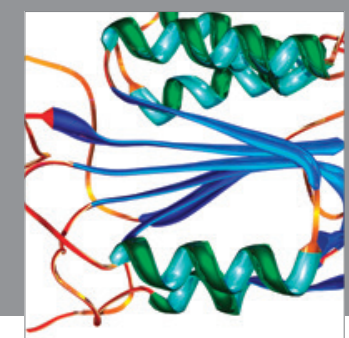

Disease Markers
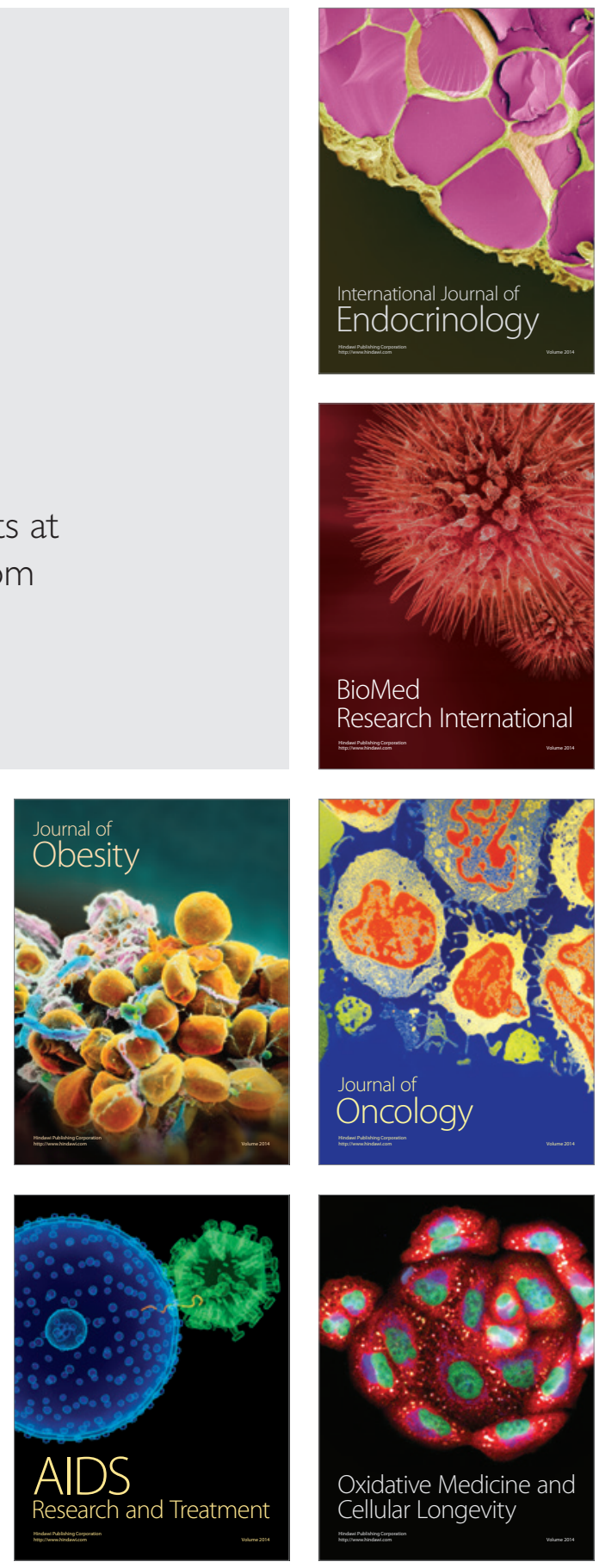